Article

\title{
Identification of Major Flavone $C$-Glycosides and Their Optimized Extraction from Cymbidium kanran Using Deep Eutectic Solvents
}

\author{
Kyung Min Jeong ${ }^{1}$, Misuk Yang ${ }^{2}$, Yan Jin ${ }^{1}$, Eun Mi Kim ${ }^{1}$, Jaeyoung Ko ${ }^{2, *}$ and \\ Jeongmi Lee ${ }^{1, *}$ \\ 1 School of Pharmacy, Sungkyunkwan University, Jangan-gu, Suwon 16419, Gyeonggi-do, Korea; \\ aeroserp86@naver.com (K.M.J.); kimyeon909@gmail.com (Y.J.); em131632@gmail.com (E.M.K.) \\ 2 Amorepacific Research and Development Center, Giheung-gu, Yongin 17074, Gyeonggi-do, Korea; \\ msyang@amorepacific.com \\ * Correspondence: jaeyoungko@amorepacific.com (J.K.); jlee0610@skku.edu (J.L.); \\ Tel.: +82-31-280-5928 (J.K.); +82-31-290-7784 (J.L.)
}

Received: 30 October 2017; Accepted: 16 November 2017; Published: 18 November 2017

\begin{abstract}
Cymbidium kanran, an orchid exclusively distributed in Northeast Asia, has been highly valued as a decorative plant and traditional herbal medicine. Here, C. kanran extracts were prepared in $70 \%$ aqueous methanol using ultrasound-assisted extraction (UAE) and subjected to liquid chromatography-photodiode array detection and ultra-high performance liquid chromatography-quadrupole-time-of-flight-mass spectrometry analysis, which were used for quantitative and qualitative analysis, respectively. It was found that the extracts were rich in flavone C-glycosides including vicenin-2, vicenin-3, schaftoside, vitexin, and isovitexin. Ten deep eutectic solvents (DESs) were synthesized by combining choline chloride (hydrogen bond acceptor) with various polyols and diols (hydrogen bond donors) and were tested as a medium for the efficient production of extracts enriched with potentially bioactive flavone $C$-glycosides from $C$. kanran. A DES named $\mathrm{ChCl}: \mathrm{DPG}$, composed of choline chloride and dipropylene glycol at a 1:4 molar ratio, exhibited the best extraction yields. Then, the effects of extraction conditions on the extraction efficiency were investigated by response surface methodology. Lower water content in the extraction solvent and longer extraction time during UAE were desirable for higher extraction yields. Under the statistically optimized conditions, in which $100 \mathrm{mg}$ of $C$. kanran powder were extracted in $0.53 \mathrm{~mL}$ of a mixture of $\mathrm{ChCl}$ :DPG and water $(74: 26, w / w)$ for $86 \mathrm{~min}$, a total of $3.441 \mathrm{mg} \mathrm{g}^{-1}$ flavone $C$-glycosides including $1.933 \mathrm{mg} \mathrm{g}^{-1}$ vicenin-2 was obtained. This total yield was $196 \%, 131 \%$, and $71 \%$ more than those obtained using $100 \%$ methanol, water, and $70 \%$ methanol, respectively.
\end{abstract}

Keywords: Orchidaceae; Cymbidium; flavone C-glycoside profile; vicenin-2; deep eutectic solvent; response surface methodology

\section{Introduction}

Orchidaceae, commonly known as the orchid family, is a widespread family of flowering plants comprising up to 35,000 species worldwide [1]. Besides the ornamental value from their exquisite beauty, orchids have historically been recognized to have medicinal values as well [1]. The potentially bioactive phytochemicals produced by Orchidaceae cover a wide range of secondary metabolites including alkaloids, phenanthrenes, flavonoids, and floral fragrances [2]; however, comprehensive and detailed studies on their chemical and biological properties are still very limited. Cymbidium kanran is an orchid exclusively distributed in Northeast Asia including China, Japan, and Korea. C. kanran has been highly valued as a decorative plant and traditional herbal medicine with beneficial effects 
on heart, lungs, and gastroenteritis, etc. [3,4]. Chemical profiles of C. kanran have not been reported, but several flavone $C$-glycosides including vicenin-2, vitexin 7-O glucoside, and isovitexin have been found in a few Cymbidium species [5].

It is notable that flavone $C$-glycosides are the most common leaf flavonoids, followed by flavonols, in Orchidaceae [1]. Flavone $C$-glycosides, which have a sugar moiety at the C-6 or C-8 position of a flavone A ring, are stable to hydrolysis, unlike O-glycosides, and have high biological activities [6]. These have led to a gradual increase in research interest on flavone $C$-glycosides. For example, vicenin- 2 is a flavone $C$-glycoside found in diverse plants including Orchidaceae (Figure 1a) [7-9] and has shown various biological activities, including anticancer [10], anti-inflammatory [11], and antioxidant effects [12]. As a result, recent studies on vicenin-2 have focused on various aspects from the quality control of herbal medicine [13] to organic synthesis [14,15] to the treatment [10] or prevention [16] of prostate cancer.<smiles>O=c1cc(-c2ccc(O)cc2)oc2c([C@@H]3O[C@H](CO)[C@@H](O)[C@H](O)[C@H]3O)c(O)c([C@@H]3O[C@H](CO)[C@@H](O)[C@H](O)[C@H]3O)c(O)c12</smiles>

Vicenin-2<smiles>O=c1cc(-c2ccc(O)cc2)oc2c([C@@H]3OC[C@@H](O)[C@H](O)[C@H]3O)c(O)c([C@@H]3O[C@H](CO)[C@@H](O)[C@H](O)[C@H]3O)c(O)c12</smiles>

Schaftoside<smiles>O=c1cc(-c2ccc(O)cc2)oc2c(C3OC[C@@H](O)[C@H](O)[C@H]3O)c(O)c([C@@H]3O[C@H](CO)[C@@H](O)[C@H](O)[C@H]3O)c(O)c12</smiles>

Vicenin-3<smiles>O=c1cc(-c2ccc(O)cc2)oc2c([C@H]3O[C@H](CO)[C@@H](O)[C@H](O)[C@H]3O)c(O)cc(O)c12</smiles>

Vitexin<smiles>O=c1cc(-c2ccc(O)cc2)oc2cc(O)c([C@@H]3O[C@H](CO)[C@@H](O)[C@H](O)[C@H]3O)c(O)c12</smiles>

(a)

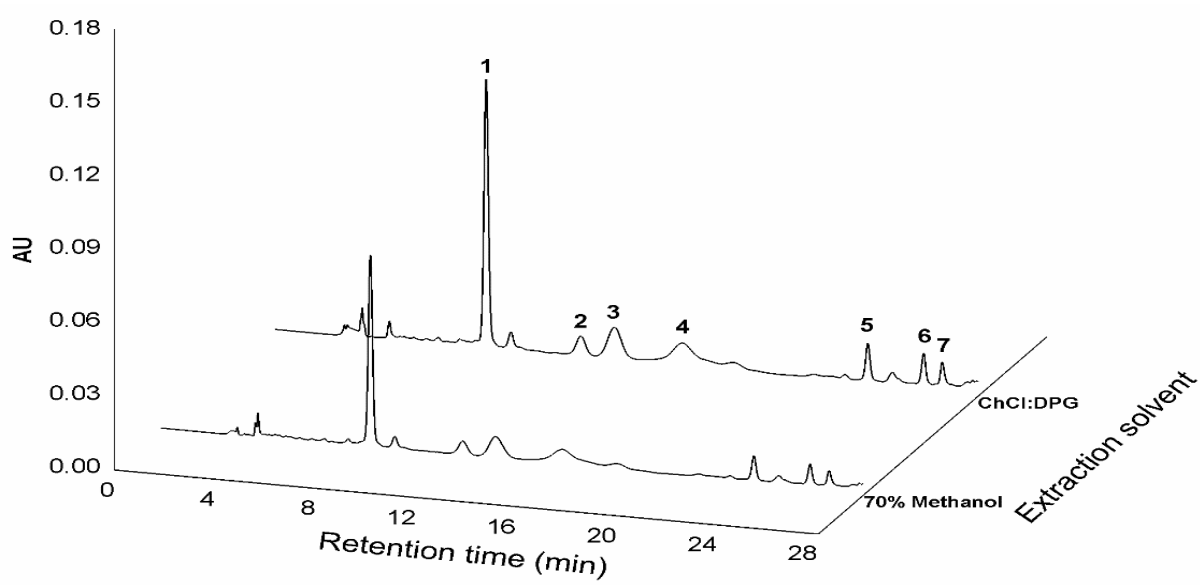

(b)

Figure 1. Chemical structures of the flavone C-glycosides in C. kanran identified in this study (a) and chromatograms of the C. kanran extracts (b). Peak identification: 1, vicenin-2; 2, vicenin-2 isomer; 3 , schaftoside isomer; 4 , schaftoside; 5 , vicenin-3; 6 , vitexin; 7 , isovitexin. 
For the chemical characterization and recovery of bioactive compounds from plants, organic solvents with diverse polarities are usually used as the dissolution and extraction media [17]. In particular, polar organic solvents or their aqueous solutions are often used for flavonoid extraction. In conformity with the needs of modern chemistry, replacement of the conventional organic solvents by ecofriendly and safe solvents has been increasingly demanded [18]. In this regard, deep eutectic solvents (DESs) have been suggested as a desirable alternative [19]. DESs are a fluid system composed of two or more components associated via hydrogen bond interactions with a melting point far below either of the individual components [20]. The hydrogen-bond donors (HBDs) include amides, carboxylic acids, alcohols, sugars, and sugar alcohols, while the hydrogen-bond acceptors (HBAs) are usually quaternary ammonium salts. Due to their safety and high solubilization ability regardless of polarity, DESs could serve as a desirable extraction medium [21]. Extraction techniques are also an important factor affecting extraction efficiency. For polyphenol and flavonoid extraction, various extraction methods have been used including heat reflux extraction, microwave-assisted extraction, pressurized liquid extraction, and ultrasound-assisted extraction (UAE) [22]. In particular, UAE is preferable for thermally labile compounds [23] and offers high extraction efficiency by facilitating solvent penetration and tissue disruption [24,25].

The aim of this study was to acquire green extracts of $C$. kanran enriched with potentially bioactive compounds at maximized efficiency. For this, first, the chemical profile of flavone $C$-glycosides in $C$. kanran was investigated. Then, a series of DESs were logically designed and synthesized, and a DES with the highest extraction efficiency was sought. Finally, the effects of the extraction conditions based on UAE on the extraction efficiency were assessed, followed by the optimization of the extraction conditions using response surface methodology (RSM).

\section{Results and Discussion}

\subsection{Chemical Profiling of C. kanran Extracts}

For the chemical profiling of C. kanran, its extracts were initially prepared in $70 \%$ methanol by UAE, which has been suggested as a useful tool to extract or recover bioactive compounds from various sources [26-28]. Liquid chromatography-photodiode array (LC-PDA) analysis of the extracts displayed a number of peaks having two $\lambda_{\max }$ values at $\sim 270 \mathrm{~nm}$ and $\sim 335 \mathrm{~nm}$ between 8 and $28 \mathrm{~min}$ (Supplementary Materials Figure S1 and Figure 1b). This type of absorption spectrum is indicative of flavone structures [29]. The weak elution conditions for these peaks indicated that they were likely to be glycosides. Subsequent analysis using ultra-high performance liquid chromatography-quadrupole-time-of-flight mass spectrometry allowed the identification of seven flavone C-glycosides (Table 1). The chromatographic peaks 1, 4, 5, 6, and 7 in Figure 1 were confirmed to be vicenin-2, schaftoside, vicenin-3, vitexin, and isovitexin, respectively. Peaks 2 and 3 exhibited the same exact mass values and UV spectra as vicenin-2 and schaftoside, respectively (Table 1), but could not be identified due to difficulty in standard procurement for the candidate compounds. Accordingly, peaks 2 and 3 were putatively named as a vicenin- 2 isomer and schaftoside isomer, respectively, and the subsequent studies were conducted by quantifying the seven flavone $C$-glycosides. This is the first report of chemical profiles of flavone $C$-glycosides in a Cymbidium species. In addition, the developed LC-PDA method enabled the simultaneous determination of major flavone C-glycosides in C. kanran. 
Table 1. Identification of the major flavone C-glycosides in C. kanran.

\begin{tabular}{|c|c|c|c|c|c|c|c|c|c|c|}
\hline \multirow{2}{*}{ No. ${ }^{a}$} & \multirow{2}{*}{ Compound } & \multirow{2}{*}{$\lambda_{\max } b$} & \multirow{2}{*}{$\mathrm{MW}^{\mathrm{c}}$} & \multirow{2}{*}{ Molecular Formula } & \multicolumn{2}{|c|}{ Theoretical } & \multicolumn{2}{|c|}{ Measured } & \multicolumn{2}{|c|}{ Mass Error ${ }^{d}$} \\
\hline & & & & & {$[\mathbf{M}+\mathbf{H}]^{+}$} & {$[\mathbf{M}-\mathbf{H}]^{-}$} & {$[\mathbf{M}+\mathbf{H}]^{+}$} & {$[\mathbf{M}-\mathbf{H}]^{-}$} & {$[\mathbf{M}+\mathbf{H}]^{+}$} & {$[\mathbf{M}-\mathbf{H}]^{-}$} \\
\hline 1 & Vicenin-2 & 334 & 594.1584 & $\mathrm{C}_{27} \mathrm{H}_{30} \mathrm{O}_{15}$ & 595.1663 & 593.1506 & 595.1655 & 593.1509 & -1.3 & 0.5 \\
\hline 2 & Vicenin-2 isomer & 333 & 594.1584 & $\mathrm{C}_{27} \mathrm{H}_{30} \mathrm{O}_{15}$ & 595.1663 & 593.1506 & 595.1658 & 593.1501 & -0.8 & -0.8 \\
\hline 3 & Schaftoside isomer & 335 & 564.1479 & $\mathrm{C}_{26} \mathrm{H}_{28} \mathrm{O}_{14}$ & 565.1557 & 563.1401 & 565.1546 & 563.1392 & -1.9 & -1.6 \\
\hline 4 & Schaftoside & 336 & 564.1479 & $\mathrm{C}_{26} \mathrm{H}_{28} \mathrm{O}_{14}$ & 565.1557 & 563.1401 & 565.1542 & 563.1411 & -2.7 & 1.8 \\
\hline 5 & Vicenin-3 & 334 & 564.1479 & $\mathrm{C}_{26} \mathrm{H}_{28} \mathrm{O}_{14}$ & 565.1557 & 563.1401 & 565.1541 & 563.1404 & -2.8 & 0.5 \\
\hline 6 & Vitexin & 336 & 432.1056 & $\mathrm{C}_{21} \mathrm{H}_{20} \mathrm{O}_{10}$ & 433.1135 & 431.0978 & 433.1136 & 431.0976 & 0.2 & -0.5 \\
\hline 7 & Isovitexin & 336 & 432.1056 & $\mathrm{C}_{21} \mathrm{H}_{20} \mathrm{O}_{10}$ & 433.1135 & 431.0978 & 433.1122 & 431.0981 & -3.0 & 0.7 \\
\hline
\end{tabular}

${ }^{\mathbf{a}}$ Peak identification number in Figure $1 ;^{\mathbf{b}} \mathrm{nm} ;^{\mathbf{c}} \mathrm{g} \mathrm{mol}{ }^{-1} ;{ }^{\mathbf{d}} \mathrm{ppm}$. 


\subsection{Preparation of a Series of Deep Eutectic Solvents}

Numerous combinations of HBAs and HBDs from renewable, inexpensive, and readily accessible resources are possible [30]. In this study, the HBA was fixed as choline chloride, which usually permits easy synthesis of DESs and therefore is the most common component of DESs [31]. For its counterpart, diol and polyol types were selected as HBDs, because a series of diols and polyols with similar chemical structures were available and because diol- or polyol-based DESs were less viscous than DESs containing carboxylic acids or sugars, therefore easing the solvent handling [20].

Specifically, four polyol compounds, from glycerol to maltitol with an increasing number of alcohols, and six diol compounds, from 1,2-ethanediol to 1,6-hexanediol and dipropylene glycol with an increasing carbon chain length, were selected (Table 2). All of the HBD components could form DESs with choline chloride at the ratios described in Table 2 by the heating method, and the resulting solvents are listed in Table 2.

Table 2. List of the choline chloride-based deep eutectic solvents (DESs) synthesized in this study.

\begin{tabular}{cccc}
\hline Abbreviation & Hydrogen Bond Acceptor & Hydrogen Bond Donor & Molar Ratio \\
\hline ChCl:Gly & & Glycerol & $1: 4$ \\
ChCl:Xyl & Xylitol & $1: 1$ \\
ChCl:Sor & D-sorbitol & $1: 1$ \\
ChCl:Mal & Maltitol & $1: 1$ \\
ChCl:Eth & Choline chloride & 1,2-Ethanediol & $1: 4$ \\
ChCl:Prop & & 1,3-Propanediol & $1: 4$ \\
ChCl:But & 1,4-Butanediol & $1: 4$ \\
ChCl:Pent & 1,5-Pentanediol & $1: 4$ \\
ChCl:Hex & 1,6-Hexanediol & $1: 4$ \\
ChCl:DPG & Dipropylene glycol & $1: 4$ \\
\hline
\end{tabular}

\subsection{Selection of Deep Eutectic Solvents with High Extraction Efficiency}

Although UAE has been very effective with the use of DESs [32,33], the possibility remained that other extraction methods might work better in this study. Thus, three simple extraction methods easily compatible with DESs were tested for comparison including stirring, heating, and heating with stirring methods. As shown in Figure S2, the three methods tested did not differ from each other but were significantly inferior to UAE based on the total extracted amounts of flavone $C$-glycosides. Therefore, UAE was maintained as the extraction method in the following experiments.

Vicenin-2 was predominant among the seven flavone $C$-glycosides in all of the extracts prepared in various solvents (Figure S3). The total yields of aqueous mixtures of organic solvents were higher than those of pure methanol or ethanol, in which yields were much lower than that of water (Figure S3). These results show that the extraction of flavonoid glycosides having both non-polar and polar moieties was favored when using organic solvents with reasonable polarity.

Table 3 shows that the extraction efficiencies were greatly affected by the DES types, that is, the HBD components. Among the polyol-based DESs, the extraction yields increased as the number of hydroxyl groups of the HBD decreased. In the case of the diol-based DESs, the yields steadily increased with the increasing carbon chain length, e.g., from $C_{2}$ to $C_{6}$. In both cases, the enhanced extraction yields were probably due to the increased hydrophobicity of the HBDs in the DESs. Similar observations have been reported on the effects of carbon length of the cation of ionic liquids on the extraction efficiency [34].

The two diol-based DESs, ChCl:Hex and $\mathrm{ChCl}$ :DPG, included 1,6-hexanediol and dipropylene glycol, respectively, as the HBDs. They displayed the highest extraction yields. Although the differences between them were not significant, the latter was selected as the final extraction solvent because it exhibited slightly higher values than the former. 


\subsection{Effects of Variables on the Extraction Efficiency for Flavone C-Glycosides}

The most important categorical variable influencing the extraction efficiency could be the extraction solvent, which was selected to be ChCl:DPG, as discussed in Section 2.3. Several numerical variables that could be influential are the extraction time, ratio of solvent to sample solids, and water content in the extraction solvent. Therefore, the following three variables were included in a central composite design (CCD) for RSM analysis: $(A)$ ultrasonic irradiation time; $(B)$ volume of extraction solvent per $100 \mathrm{mg}$ of $C$. kanran powder; and $(C)$ content of water in the extraction solvent. They were varied at five levels $(-\alpha,-1,0,+1,+\alpha)$, and the real ranges tested were as follows: $A, 0.3-90 \mathrm{~min}$; $B, 0.5-1.75 \mathrm{~mL} ; \mathrm{C}, 1.6-100 \% \mathrm{w} / \mathrm{w}$.

The experiments were conducted in random order and showed that the extracted amounts of the individual compounds and their summed amounts varied in a very similar fashion depending on the experimental conditions. Accordingly, the total yields of seven flavone $C$-glycosides were input as the response $(Y)$, which yielded the following polynomial quadratic equation in coded values:

$$
Y=2.34+0.15 A+0.082 \mathrm{~B}-0.29 \mathrm{C}-0.28 \mathrm{AB}+0.22 \mathrm{AC}+0.15 \mathrm{BC}+0.071 A^{2}+0.090 B^{2}-0.28 C^{2}
$$

The model evaluation results based on the analysis of variance (ANOVA) are summarized in Table S1. All of the figures of merit indicated that the established model is valid.

The equation above and the ANOVA results show that the water content in the extraction solvent $\left(C\right.$ and $\left.C^{2}\right)$ was the most influential variable in a negative fashion $(p<0.05)$. That is, lower water contents were preferable for higher extraction yields. This is somewhat consistent with the above observations that the extraction efficiency for flavone $C$-glycosides tended to improve with decreasing polarity of the HBD components.

In contrast, the extraction time $(A)$ had a significant influence in a positive direction $(p<0.05)$, indicating that a longer extraction time could lead to higher yields. The necessity for a longer irradiation time than in previous studies where leaves or skins were used $[32,33]$ might be attributed to the native physical properties of $C$. kanran samples that contained dense tissues such as stalks and roots from the whole plants. The solid-to-liquid ratio $(B)$ showed no significant influence. Additionally, information on the interactive effects could be also obtained. A negative interaction was observed between the extraction time and the extractant volume $(p<0.05)$, while the time and the water content had a positive interaction $(p<0.05)$.

\subsection{Optimization of the Extraction Conditions}

A solution was sought for the optimal conditions that could lead to the maximal extraction yields. The resulting conditions involved the extraction of $100 \mathrm{mg}$ of sample powder in $0.53 \mathrm{~mL}$ of a mixture of $\mathrm{ChCl}$ :DPG and water $(74: 26, w / w)$ for $86 \mathrm{~min}$ of ultrasonic irradiation. When the extraction experiment was actually performed under the optimized conditions, a total yield of $3.441 \mathrm{mg} \mathrm{g}^{-1}$ flavone $C$-glycosides ( $\mathrm{n}=3,8.46 \% \mathrm{RSD})$ was measured, and this was very close to the predicted yield of $3.49 \mathrm{mg} \mathrm{g}^{-1}$. Among the total flavone $C$-glycosides extracted was vicenin-2, present at $56 \%$ $\left(1.933 \mathrm{mg} \mathrm{g}^{-1}\right)$. This total yield is almost as thrice that obtained using $100 \%$ methanol and $71 \%$ more than the yield obtained using $70 \%$ methanol (Figure $1 \mathrm{~b}$ and Table 3 ). 
Table 3. Extraction yields for flavone $C$-glycosides of the tested solvents.

\begin{tabular}{|c|c|c|c|c|c|c|c|c|}
\hline \multirow{2}{*}{ Extraction Solvent } & \multicolumn{8}{|c|}{ Extracted Amount $^{\text {a }}($ Mean $\pm S D, n=3)$} \\
\hline & Vicenin-2 & Vicenin-2 Isomer & Schaftoside Isomer & Schaftoside & Vicenin-3 & Vitexin & Isovitexin & Summed Amount \\
\hline Water & $0.940( \pm 0.002)$ & $0.115( \pm 0.002)$ & $0.157( \pm 0.007)$ & $0.174( \pm 0.007)$ & $0.072( \pm 0.002)$ & $0.023( \pm 0.002)$ & $0.005( \pm 0.000)$ & $1.486^{* * *, b}( \pm 0.016)$ \\
\hline 100\% Methanol & $0.623( \pm 0.022)$ & $0.090( \pm 0.003)$ & $0.156( \pm 0.004)$ & $0.150( \pm 0.002)$ & $0.066( \pm 0.001)$ & $0.048( \pm 0.001)$ & $0.025( \pm 0.000)$ & $1.158^{* * *}( \pm 0.034)$ \\
\hline $70 \%$ Methanol & $1.130( \pm 0.006)$ & $0.158( \pm 0.006)$ & $0.262( \pm 0.007)$ & $0.258( \pm 0.012)$ & $0.107( \pm 0.001)$ & $0.057( \pm 0.001)$ & $0.029( \pm 0.000)$ & $2.001^{* * *}( \pm 0.017)$ \\
\hline $70 \%$ Ethanol & $1.186( \pm 0.015)$ & $0.169( \pm 0.002)$ & $0.281( \pm 0.006)$ & $0.264( \pm 0.009)$ & $0.116( \pm 0.001)$ & $0.060( \pm 0.000)$ & $0.031( \pm 0.000)$ & $2.107^{* * *}( \pm 0.019)$ \\
\hline ChCl:Gly & $1.153( \pm 0.061)$ & $0.151( \pm 0.001)$ & $0.258( \pm 0.010)$ & $0.225( \pm 0.005)$ & $0.102( \pm 0.006)$ & $0.052( \pm 0.004)$ & $0.025( \pm 0.002)$ & $1.966^{* * *}( \pm 0.092)$ \\
\hline ChCl:Xyl & $0.964( \pm 0.031)$ & $0.127( \pm 0.005)$ & $0.208( \pm 0.003)$ & $0.192( \pm 0.004)$ & $0.082( \pm 0.002)$ & $0.041( \pm 0.001)$ & $0.019( \pm 0.000)$ & $1.633^{* * *}( \pm 0.040)$ \\
\hline ChCl:Sor & $0.859( \pm 0.033)$ & $0.111( \pm 0.010)$ & $0.178( \pm 0.012)$ & $0.164( \pm 0.015)$ & $0.073( \pm 0.004)$ & $0.035( \pm 0.002)$ & $0.015( \pm 0.000)$ & $1.435^{* * *}( \pm 0.075)$ \\
\hline ChCl:Mal & $0.774( \pm 0.062)$ & $0.093( \pm 0.004)$ & $0.162( \pm 0.008)$ & $0.144( \pm 0.018)$ & $0.063( \pm 0.004)$ & $0.031( \pm 0.001)$ & $0.014( \pm 0.000)$ & $1.281^{* * *}( \pm 0.085)$ \\
\hline ChCl:Eth & $1.210( \pm 0.068)$ & $0.161( \pm 0.006)$ & $0.244( \pm 0.002)$ & $0.219( \pm 0.002)$ & $0.110( \pm 0.006)$ & $0.057( \pm 0.003)$ & $0.027( \pm 0.000)$ & $2.028^{* * *}( \pm 0.086)$ \\
\hline ChCl:Prop & $1.275( \pm 0.121)$ & $0.175( \pm 0.017)$ & $0.288( \pm 0.027)$ & $0.272( \pm 0.034)$ & $0.120( \pm 0.010)$ & $0.065( \pm 0.005)$ & $0.032( \pm 0.003)$ & $2.227^{* * *}( \pm 0.220)$ \\
\hline ChCl:But & $1.324( \pm 0.104)$ & $0.184( \pm 0.012)$ & $0.298( \pm 0.025)$ & $0.283( \pm 0.029)$ & $0.128( \pm 0.011)$ & $0.069( \pm 0.006)$ & $0.034( \pm 0.003)$ & $2.320^{* * *}( \pm 0.194)$ \\
\hline ChCl:Pent & $1.460( \pm 0.058)$ & $0.193( \pm 0.002)$ & $0.328( \pm 0.001)$ & $0.296( \pm 0.021)$ & $0.141( \pm 0.006)$ & $0.076( \pm 0.002)$ & $0.040( \pm 0.002)$ & $2.534 * *( \pm 0.049)$ \\
\hline ChCl:Hex & $1.533( \pm 0.009)$ & $0.197( \pm 0.013)$ & $0.339( \pm 0.006)$ & $0.325( \pm 0.005)$ & $0.151( \pm 0.002)$ & $0.079( \pm 0.001)$ & $0.040( \pm 0.000)$ & $2.664( \pm 0.004)$ \\
\hline ChCl:DPG & $1.652( \pm 0.052)$ & $0.233( \pm 0.003)$ & $0.370( \pm 0.021)$ & $0.347( \pm 0.026)$ & $0.162( \pm 0.005)$ & $0.087( \pm 0.003)$ & $0.045( \pm 0.000)$ & $2.896( \pm 0.108)$ \\
\hline Optimized conditions & $1.933( \pm 0.158)$ & $0.277( \pm 0.025)$ & $0.441( \pm 0.039)$ & $0.452( \pm 0.039)$ & $0.188( \pm 0.014)$ & $0.100( \pm 0.010)$ & $0.050( \pm 0.004)$ & $3.441( \pm 0.291)$ \\
\hline
\end{tabular}

${ }^{\mathrm{a}} \mathrm{mg} \mathrm{g}^{-1}{ }^{\mathrm{b}}$ statistical difference in comparison to ChCl:DPG was indicated with ${ }^{* *}(p<0.01)$ and ${ }^{* * *}(p<0.001)$. 


\section{Materials and Methods}

\subsection{Chemicals, Reagents, and Equipment}

Whole dried C. kanran that had been cultivated in Jeju, Korea was provided by Amorepacific Corporation (Seoul, Korea). They were finely pulverized and kept at $-20^{\circ} \mathrm{C}$ until analysis. Compounds used for DES preparation are listed in Table 2, and their detailed information is summarized in Table S2. Analytical standards including vicenin-2 ( $\geq 98.0 \%)$, schaftoside $(\geq 98.0 \%)$, and vicenin-3 $(\geq 98.0 \%)$ were obtained from Alfa Biotechnology Co., Ltd. (Chengdu, Sichuan, China), while vitexin (95.0\%) and isovitexin (98.0\%) were purchased from Sigma-Aldrich (St. Louis, MO, USA). Formic acid and trifluoroacetic acid were also from Sigma-Aldrich. HPLC-grade acetonitrile and water were obtained from J.T. Baker (Phillipsburg, NJ, USA).

An ultrasonic bath (Powersonic 410) and a heating magnetic stirrer were obtained from Hwashin Technology (Seoul, Korea) and VELP Scientifica (Usmate, Italy), respectively.

\subsection{Preparation of Analytical Standard Solutions and Deep Eutectic Solvents}

Each stock solution of vicenin-2, schaftoside, vicenin-3, vitexin, and isovitexin was prepared in methanol at $1 \mathrm{mg} \mathrm{mL}^{-1}$ and stored at $-20{ }^{\circ} \mathrm{C}$. Standard working solutions were produced in methanol at final concentrations of $2.5,5,10,20,40,50$, and $80 \mu \mathrm{g} \mathrm{mL}{ }^{-1}$ for vicenin-2; $0.5,1,2,4,8,10$, and $16 \mu \mathrm{g} \mathrm{mL}^{-1}$ for schaftoside; $0.5,1,2,4,8$, and $10 \mu \mathrm{g} \mathrm{mL}^{-1}$ for vicenin-3 and vitexin; and $0.25,0.5,1$, 2,4 , and $5 \mu \mathrm{g} \mathrm{mL}^{-1}$ for isovitexin. These were used for validation of the quantitative method assessing linearity, precision, and accuracy. DESs were synthesized using a heating method as previously described [32]. For the solvent selection procedure, DESs were mixed with water to produce $85 \% w / w$ aqueous mixtures for easy handling [35].

\subsection{Ultra-High Performance Liquid Chromatography-Quadrupole-Time-of-Flight Mass Spectrometry Analysis of C. kanran Extracts}

The experimental conditions were slightly modified from a previous study [36]. The stationary phase was an ACQUITY UPLC BEH C18 column $(50 \mathrm{~mm} \times 2.1 \mathrm{~mm}, 1.7 \mu \mathrm{m})$ from Waters (Milford, MA, USA), and a linear gradient elution was conducted using a mobile phase consisting of (A) $0.1 \%$ formic acid in water and (B) $0.1 \%$ formic acid in acetonitrile: $0-5 \mathrm{~min}, 1-10 \% \mathrm{~B} ; 5-8 \mathrm{~min}, 10-25 \% \mathrm{~B}$; 8-10 $\min 25-45 \%$ B; 10-16 min 45-75\% B; 16-18 min, 75-100\% B; 18-21 min, 100\% B. Parameters of the mass spectrometer were set as follows: capillary voltage, $1.9 \mathrm{kV}$; sample cone, $40 \mathrm{~V}$; source temperature, $100{ }^{\circ} \mathrm{C}$; desolvation gas (nitrogen), $1000 \mathrm{~L} \mathrm{~h}^{-1}$. The high collision energy ramps ranged from 10 to $20 \mathrm{~V}$. Samples were diluted 10-fold with water and filtered through a 0.2- $\mu$ m membrane filter (Whatman, Piscataway, NJ, USA) before injection.

\subsection{Liquid Chromatography-Photodiode Array Detection Analysis of C. kanran Extracts}

A Waters LC system equipped with a Waters separations module (series 2695) and a Waters photodiode array detector (series 996) was used. Empower software was used for system operation and data management. The detection wavelength was $335 \mathrm{~nm}$. Chromatographic separation was achieved on a Gemini $C_{18} 110 \AA$ column $(5 \mu \mathrm{m}, 4.6 \mathrm{~mm} \times 250 \mathrm{~mm})$ from Phenomenex (Torrance, CA, USA) at a flow rate of $1.0 \mathrm{~mL} \mathrm{~min}^{-1}$ at room temperature. The mobile phase consisted of $0.1 \%$ trifluoroacetic acid in water (A) and $0.1 \%$ trifluoroacetic acid in acetonitrile (B), and the linear gradient elution was as follows: $0-5 \mathrm{~min}, 15.5 \% \mathrm{~B} ; 5-10 \mathrm{~min}, 15.5-12.6 \% \mathrm{~B} ; 10-15 \mathrm{~min}, 12.6-13.2 \% \mathrm{~B} ; 15-20 \mathrm{~min}$, $13.2-18.5 \%$ B; $20-25 \mathrm{~min}, 18.5-23.0 \%$ B; $25-27 \mathrm{~min}, 23.0 \%$ B; $27-28 \mathrm{~min}, 23.0-100.0 \%$ B. The system was returned to the initial conditions and equilibrated for $10 \mathrm{~min}$ before subsequent injections. Samples diluted in water were membrane-filtered prior to injection.

The quantification method was validated as previously described [32]. The calibration curves for vicenin-2, schaftoside, vicenin-3, vitexin, and isovitexin were linear $\left(\mathrm{r}^{2} \geq 0.9963\right)$, precise 
$(\leq 12.6 \% \mathrm{RSD})$, and accurate $(90.8-112.6 \%)$ within the tested ranges (please see supplementary materials for detailed results).

\subsection{Extraction of Flavone C-Glycosides from C. kanran}

The following conditions were used for UAE before the extraction conditions were optimized. One hundred $\mathrm{mg}$ of the dried C. kanran powder were mixed in $1 \mathrm{~mL}$ of extraction solvent (water, methanol, ethanol, $70 \%$ aqueous methanol, $70 \%$ aqueous ethanol, or DESs). After being vortexed briefly, the mixture was irradiated at ambient temperature under the maximum power $(\sim 500 \mathrm{~W})$ without thermal control for $45 \mathrm{~min}$. The bath temperature tended to rise with increasing irradiation time; it was approximately $40{ }^{\circ} \mathrm{C}$ after extraction. After centrifugation at $12,300 \times g$ for $20 \mathrm{~min}$, the cleared supernatant was removed and diluted with water for chromatographic analysis. After condition optimization, the extraction was conducted under the conditions described in Section 2.5.

Stirring, heating, and heating with stirring methods were tested in comparison to the UAE method as follows: $100 \mathrm{mg}$ of $C$. kanran powder were extracted in $1 \mathrm{~mL}$ of $70 \%$ methanol for $45 \mathrm{~min}$ by stirring (600 rpm, room temperature), heating $\left(60^{\circ} \mathrm{C}\right)$, or heating with stirring $\left(600 \mathrm{rpm}, 60^{\circ} \mathrm{C}\right)$. Each extract was analyzed by LC-PDA.

Extraction yields were expressed as the individual or total amount $(\mathrm{mg})$ of extracted flavone C-glycosides per mass (g) of C. kanran powder $\left(\mathrm{mg} \mathrm{g}^{-1}\right)$.

\subsection{Experimental Design and Statistical Analysis}

RSM based on a CCD was conducted using the Design-Expert Ver. 8.0 (Statease Inc., Minneapolis, MN, USA). Further statistical analysis was performed using GraphPad Prism 5.01 for Windows (GraphPad Software, San Diego, CA, USA) as previously described [32].

\section{Conclusions}

In this study, the chemical profile of C. kanran was investigated for the first time, and it was found that $C$. kanran is rich in flavone $C$-glycosides, especially vicenin-2, the most prevalent one that comprised more than $50 \%$ of the total flavone C-glycosides. Ten different DESs were logically designed by varying either the number of alcohol groups in polyol type solvents or the length of the carbon chain in diol type solvents. The solvent extraction efficiency for the flavone $C$-glycosides improved with fewer alcohol groups or longer carbon chain lengths in the HBD component, which is probably because the non-polar property from the HBD compartment was needed for efficient interaction with the hydrophobic flavone moiety. The RSM-based investigation of several variables for the extraction suggested that lower water content in the extraction solvent and longer extraction time during UAE were desirable for higher extraction yields. The final optimized conditions resulted in the extraction of a total of $3.441 \mathrm{mg} \mathrm{g}^{-1}$ flavone $C$-glycosides including $1.933 \mathrm{mg} \mathrm{g}^{-1}$ vicenin-2; this total yield is $196 \%$, $131 \%$, and $71 \%$ more than those obtained using $100 \%$ methanol, water, and $70 \%$ methanol, respectively. This study showed that the potentially bioactive flavone $C$-glycosides including vicenin-2 could be extracted in DES with maximal efficiency under the optimized conditions.

Supplementary Materials: The following are available online. Results S1: Validation results of the LC-PDA method for flavone $C$-glycosides; Figure S1: a two dimensional chromatogram from the LC-PDA analysis of $C$. kanran extracts obtained in 70\% aqueous methanol; Figure S2: extraction efficiency of heating, stirring, heating with stirring, and UAE methods using 70\% aqueous methanol; Figure S3: overlaid chromatograms of the C. kanran extracts obtained in water, methanol, ethanol, 70\% methanol, 70\% ethanol, and 10 different DESs. Table S1: ANOVA results of the established model; Table S2: compounds used for the preparation of deep eutectic solvents.

Acknowledgments: This research was supported by a grant from the Amorepacific Corporation (grant No. ORT22-01-R17E999001) within the Amorepacific Open Research Program.

Author Contributions: J.K., K.M.J., and J.L. conceived and designed the experiments; K.M.J., J.K., M.Y., Y.J., and E.M.K. performed the experiments; J.K., M.Y., K.M.J., and J.L. analyzed the data; J.K. and M.Y. contributed materials; K.M.J. and J.L. wrote the paper.

Conflicts of Interest: The authors declare no conflict of interest. 


\section{References}

1. Hossain, M.M. Therapeutic orchids: Traditional uses and recent advances-An overview. Fitoterapia 2011, 82, 102-140. [CrossRef] [PubMed]

2. Hossain, M.M.; Kant, R.; Van, P.T.; Winarto, B.; Zeng, S.; Teixeira da Silva, J.A. The application of biotechnology to orchids. Crit. Rev. Plant Sci. 2013, 32, 69-139. [CrossRef]

3. Gutierrez, R.M.P. Orchids: A review of uses in traditional medicine, its phytochemistry and pharmacology. J. Med. Plants Res. 2010, 4, 592-638.

4. Teoh, E.S. Medicinal Orchids of Asia; Springer International Publishers: Basel, Switzerland, 2016.

5. Williams, C.A. The leaf flavonoids of the Orchidaceae. Phytochemistry 1979, 18, 803-813. [CrossRef]

6. Zeng, P.; Zhang, Y.; Pan, C.; Jia, Q.; Guo, F.; Li, Y.; Zhu, W.; Chen, K. Advances in studying of the pharmacological activities and structure-activity relationships of natural C-glycosylflavonoids. Acta Pharm. Sin. B 2013, 3, 154-162. [CrossRef]

7. Xiong, L.; Cao, Z.-X.; Peng, C.; Li, X.-H.; Xie, X.-F.; Zhang, T.-M.; Zhou, Q.-M.; Yang, L.; Guo, L. Phenolic glucosides from Dendrobium aurantiacum var. Denneanum and their bioactivities. Molecules 2013, 18, 6153-6160. [PubMed]

8. Tao, Y.; Cai, H.; Li, W.; Cai, B. Ultrafiltration coupled with high-performance liquid chromatography and quadrupole-time-of-flight mass spectrometry for screening lipase binders from different extracts of Dendrobium officinale. Anal. Bioanal. Chem. 2015, 407, 6081-6093. [CrossRef] [PubMed]

9. Ye, Z.; Dai, J.-R.; Zhang, C.-G.; Lu, Y.; Wu, L.-L.; Gong, A.G.; Xu, H.; Tsim, K.W.; Wang, Z.-T. Chemical differentiation of Dendrobium officinale and Dendrobium devonianum by using HPLC fingerprints, HPLC-ESI-MS, and HPTLC analyses. J. Evid.-Based Complement. Altern. Med. 2017, 2017. [CrossRef] [PubMed]

10. Nagaprashantha, L.D.; Vatsyayan, R.; Singhal, J.; Fast, S.; Roby, R.; Awasthi, S.; Singhal, S.S. Anti-cancer effects of novel flavonoid vicenin-2 as a single agent and in synergistic combination with docetaxel in prostate cancer. Biochem. Pharmacol. 2011, 82, 1100-1109. [CrossRef] [PubMed]

11. Kang, H.; Ku, S.K.; Jung, B.; Bae, J.S. Anti-inflammatory effects of vicenin-2 and scolymoside in vitro and in vivo. Inflamm. Res. 2015, 64, 1005-1021. [CrossRef] [PubMed]

12. Gobbo-Neto, L.; Santos, M.D.; Kanashiro, A.; Almeida, M.C.; Lucisano-Valim, Y.M.; Lopes, J.L.C.; Souza, G.E.P.; Lopes, N.P. Evaluation of the anti-inflammatory and antioxidant activities of diC-glucosylflavones from Lychnophora ericoides (Asteraceae). Planta Med. 2005, 71, 3-6. [CrossRef] [PubMed]

13. Oshima, N.; Maruyama, T.; Yamashita, T.; Uchiyama, N.; Amakura, Y.; Hyuga, S.; Hyuga, M.; Nakamori, S.; Takemoto, H.; Kobayashi, Y.; et al. Two flavone C-glycosides as quality control markers for the manufacturing process of ephedrine alkaloids-free Ephedra Herb Extract (EFE) as a crude drug preparation. J. Nat. Med. 2017. [CrossRef] [PubMed]

14. Shie, J.J.; Chen, C.A.; Lin, C.C.; Ku, A.F.; Cheng, T.J.; Fang, J.M.; Wong, C.H. Regioselective synthesis of di- C-glycosylflavones possessing anti-inflammation activities. Org. Biomol. Chem. 2010, 8, 4451-4462. [CrossRef] [PubMed]

15. Ho, T.C.; Kamimura, H.; Ohmori, K.; Suzuki, K. Total synthesis of (+)-vicenin-2. Org. Lett. 2016, 18, 4488-4490. [CrossRef] [PubMed]

16. Singhal, S.S.; Jain, D.; Singhal, P.; Awasthi, S.; Singhal, J.; Horne, D. Targeting the mercapturic acid pathway and vicenin-2 for prevention of prostate cancer. Biochim. Biophys. Acta Rev. Cancer 2017, 1868, 167-175. [CrossRef] [PubMed]

17. Herrero, M.; del Pilar Sánchez-Camargo, A.; Cifuentes, A.; Ibáñez, E. Plants, seaweeds, microalgae and food by-products as natural sources of functional ingredients obtained using pressurized liquid extraction and supercritical fluid extraction. TrAC Trends Anal. Chem. 2015, 71, 26-38. [CrossRef]

18. Ruesgas-Ramón, M.; Figueroa-Espinoza, M.C.; Durand, E. Application of deep eutectic solvents (DES) for phenolic compounds extraction: Overview, challenges, and opportunities. J. Agric. Food Chem. 2017, 65, 3591-3601. [CrossRef] [PubMed]

19. Shishov, A.; Bulatov, A.; Locatelli, M.; Carradori, S.; Andruch, V. Application of deep eutectic solvents in analytical chemistry. A review. Microchem. J. 2017, 135, 33-38. [CrossRef]

20. Zhang, Q.; Vigier, K.D.O.; Royer, S.; Jérôme, F. Deep eutectic solvents: Syntheses, properties and applications. Chem. Soc. Rev. 2012, 41, 7108-7146. [CrossRef] [PubMed] 
21. Pena-Pereira, F.; Namieśnik, J. Ionic liquids and deep eutectic mixtures: Sustainable solvents for extraction processes. ChemSusChem 2014, 7, 1784-1800. [CrossRef] [PubMed]

22. Stalikas, C.D. Extraction, separation, and detection methods for phenolic acids and flavonoids. J. Sep. Sci. 2007, 30, 3268-3295. [CrossRef] [PubMed]

23. Vilkhu, K.; Mawson, R.; Simons, L.; Bates, D. Applications and opportunities for ultrasound assisted extraction in the food industry-A review. Innov. Food Sci. Emerg. Technol. 2008, 9, 161-169. [CrossRef]

24. Huang, W.; Xue, A.; Niu, H.; Jia, Z.; Wang, J. Optimised ultrasonic-assisted extraction of flavonoids from Folium eucommiae and evaluation of antioxidant activity in multi-test systems in vitro. Food Chem. 2009, 114, 1147-1154. [CrossRef]

25. Pan, G.; Yu, G.; Zhu, C.; Qiao, J. Optimization of ultrasound-assisted extraction (UAE) of flavonoids compounds (FC) from hawthorn seed (HS). Ultrason. Sonochem. 2012, 19, 486-490. [CrossRef] [PubMed]

26. Zhu, Z.; Jiang, T.; He, J.; Barba, F.J.; Cravotto, G.; Koubaa, M. Ultrasound-assisted extraction, centrifugation and ultrafiltration: Multistage process for polyphenol recovery from purple sweet potatoes. Molecules 2016, 21, 1584. [CrossRef] [PubMed]

27. Zhua, Z.; Guan, Q.; Koubaa, M.; Barba, F.J.; Roohinejad, S.; Cravotto, G.; Yang, S.; Li, S.; He, J. HPLC-DADESI-MS 2 analytical profile of extracts obtained from purple sweet potato after green ultrasound-assisted extraction. Food Chem. 2017, 215, 391-400. [CrossRef] [PubMed]

28. Roselló-Soto, E.; Galanakis, C.M.; Brnčić, M.; Orlien, V.; Trujillo, F.J.; Mawson, R.; Knoerzer, K.; Tiwari, B.K.; Barba, F.J. Clean recovery of antioxidant compounds from plant foods, by-products and algae assisted by ultrasounds processing. Trends Food Sci. Technol. 2015, 42, 134-149. [CrossRef]

29. De Rijke, E.; Out, P.; Niessen, W.M.; Ariese, F.; Gooijer, C.; Brinkman, U.A. Analytical separation and detection methods for flavonoids. J. Chromatogr. A 2006, 1112, 31-63. [CrossRef] [PubMed]

30. Dai, Y.; van Spronsen, J.; Witkamp, G.-J.; Verpoorte, R.; Choi, Y.H. Ionic liquids and deep eutectic solvents in natural products research: Mixtures of solids as extraction solvents. J. Nat. Prod. 2013, 76, 2162-2173. [CrossRef] [PubMed]

31. Ruß, C.; König, B. Low melting mixtures in organic synthesis-An alternative to ionic liquids? Green Chem. 2012, 14, 2969-2982. [CrossRef]

32. Jeong, K.M.; Ko, J.; Zhao, J.; Jin, Y.; Yoo, D.E.; Han, S.Y.; Lee, J. Multi-functioning deep eutectic solvents as extraction and storage media for bioactive natural products that are readily applicable to cosmetic products. J. Clean. Prod. 2017, 151, 87-95. [CrossRef]

33. Jeong, K.M.; Zhao, J.; Jin, Y.; Heo, S.R.; Han, S.Y.; Yoo, D.E.; Lee, J. Highly efficient extraction of anthocyanins from grape skin using deep eutectic solvents as green and tunable media. Arch. Pharm. Res. 2015, 38, 2143-2152. [CrossRef] [PubMed]

34. Wu, K.; Zhang, Q.; Liu, Q.; Tang, F.; Long, Y.; Yao, S. Ionic liquid surfactant-mediated ultrasonic-assisted extraction coupled to HPLC: Application to analysis of tanshinones in Salvia miltiorrhiza bunge. J. Sep. Sci. 2009, 32, 4220-4226. [CrossRef] [PubMed]

35. Dai, Y.; van Spronsen, J.; Witkamp, G.-J.; Verpoorte, R.; Choi, Y.H. Natural deep eutectic solvents as new potential media for green technology. Anal. Chim. Acta 2013, 766, 61-68. [CrossRef] [PubMed]

36. Nam, M.W.; Zhao, J.; Lee, M.S.; Jeong, J.H.; Lee, J. Enhanced extraction of bioactive natural products using tailor-made deep eutectic solvents: Application to flavonoid extraction from Flos sophorae. Green Chem. 2015, 17, 1718-1727. [CrossRef]

Sample Availability: Samples of C. kanran are available from J.K. 